\title{
Disposición en la adolescencia a llevar a cabo distintas prácticas sexuales homosexuales
}

\author{
Jessica Gregori Coronado \\ al202166@uji.es \\ Carola Cerdá Salom \\ al189477@uji.es \\ MARCos LoREnZO MOYA DIAGO \\ al189538@uji.es \\ Garibaldi Perini Neto \\ al192694@uji.es \\ Rafael BAllester ARnal \\ rballest@uji.es
}

\section{Resumen}

Introducción: La orientación sexual es el deseo/atracción hacia personas del mismo o diferente sexo. Actualmente, se aconseja su evaluación a través de un continuo. Según nuestra experiencia en este campo, existe, ocasionalmente, una mayor o menor disposición a tener relaciones con personas del mismo sexo en función de una determinada práctica sexual. El objetivo de este estudio ha sido llevar a cabo un análisis de esta predisposición en población adolescente. Método: La muestra incluía 400 alumnos $(50,5 \%$ chicas y 49,5\% chicos), entre 14 y 18 años $(M=16,39$; $D T \pm 1,12)$, procedentes de distintos institutos de la Comunidad Valenciana. Los participantes cumplimentaron el cuestionario de intereses y prácticas sexuales (Unisexsida, 2014) de 5 ítems. Para este estudio se utilizaron dos ítems: orientación sexual autodefinida y disposición hacia prácticas sexuales con personas del mismo sexo. Resultados: Se observaron diferencias significativas entre chicos y chicas respecto a su disposición a dar besos en los labios, abrazos y caricias desnudos a personas del mismo sexo, siendo mayor en el caso de las adolescentes. Entre la población de adolescentes que se consideran heterosexuales, un $4,5 \%$ y un $59,9 \%$ se encuentra dispuesto a realizar ciertas prácticas con personas del mismo sexo. En esta población existen diferencias significativas entre chicas y chicos respecto a su disposición a dar besos en los labios, abrazos y caricias desnudos y dejarse masturbar por personas de su mismo sexo. Conclusiones: Estos resultados enfatizan la necesidad de dejar de considerar la orientación sexual como una categoría estanca, de manera que refleje la diversidad afectivo-sexual.

Palabras clave: orientación sexual, adolescencia, prácticas sexuales, predisposición sexual, diversidad afectivo-sexual. 


\section{Abstract}

Introduction: Sexual orientation is defined as the desire/sexual attraction to people of the same or opposite sex. Currently, its evaluation is advised through a continuum. According to our experience in this field, there is occasionally more or less willingness to have sex with the same sex depending on a particular sexual practice. The aim of this study was to analyse this predisposition in an adolescent population. Method: The sample included 400 students $(50.5 \%$ female, $49.5 \%$ male), aged $14-18$ years $(M=16.39$; $\mathrm{SD} \pm 1.12$ ), from different high schools in the Valencian Community (Spain). Participants completed the questionnaire Sexual Interests and Practices (Unisexsida, 2014) with five items, although only the first (self-reported sexual orientation) and the last (disposition towards different sexual practices with the same sex) items were used. Results: Significant gender differences were found in their willingness to kiss on the lips or to hug and caress naked people of the same sex, which were higher for females. In the adolescent population who considered themselves heterosexuals, from $4.5 \%$ to $59.9 \%$ were willing to undertake certain practices with persons of their same sex. Significant gender differences were also found in this same population as to their willingness to kiss on the lips, hug and caress, and be masturbated by, persons of the same sex. Conclusions: These results stress the need to stop considering sexual orientation as a tight category to reflect sexual diversity.

Keywords: sexual orientation, adolescence, sexual practices, sexual predisposition, sexual diversity.

\section{Introducción}

Actualmente, el término orientación sexual se define como una atracción emocional, romántica, sexual o afectiva duradera hacia otros. Esta se da a lo largo de un continuo, que abarca desde la homosexualidad exclusiva hasta la heterosexualidad absoluta, e incluye diversas formas de bisexualidad (APA, 2013).

Respecto a la evolución de este concepto, Kinsey (1948) propuso un modelo, tras llevar a cabo un estudio de prevalencia de la homosexualidad/bisexualidad en la sociedad y de los deseos/experiencias sexuales homosexuales en población heterosexual, según el cual no existen dos categorías excluyentes de orientación sexual, sino que se trata de un continuo donde un individuo se puede situar en cualquier punto desde «exclusivamente heterosexual» hasta «exclusivamente homosexual», encontrándose la bisexualidad en el centro del continuo.

En 1985, Klein aborda las deficiencias de Kinsey y propone un modelo en el que la vivencia de la orientación sexual se explica por siete factores que pueden cambiar a lo largo del tiempo (pasado, presente y futuro idealizado): atracción sexual, comportamiento sexual, fantasías sexuales, preferencia emocional, preferencia social, estilo de vida heterosexual/homosexual y autoidentificación.

En las últimas décadas, la investigación acerca de la prevalencia de las identidades lesbianas, bisexuales y gays (LGB) y de las actitudes hacia estas distintas orientaciones sexuales en la población adolescente está obteniendo mayor relevancia y consciencia social, ya que permite comprender la influencia de los diferentes agentes de socialización en la tolerancia 
hacia la diversidad sexual y, consecuentemente, utilizar estrategias que permitan el desarrollo de actitudes más positivas.

En la actualidad, España se encuentra entre los países más avanzados respecto a la regulación legal de la igualdad en las distintas orientaciones sexuales. Sin embargo, existen otros países como Irán, Mauritania, Arabia Saudí, Sudán, los Emiratos Árabes Unidos, Yemen y Nigeria, donde las identidades que transgreden el rígido modelo dimórfico de heterosexualidad son duramente castigadas y patologizadas (Grant, Mottet, Tanis, Herman y Keisling, 2011).

En muchos de estos países el código penal no se aplica sistemáticamente, pero su mera existencia refuerza una cultura donde una parte significativa de la población necesita esconderse por miedo a las represalias y este hecho permite la no aceptación de la propia orientación sexual como homosexuales y la no realización de prácticas sexuales con personas del mismo sexo aunque se desee (Jäckle y Wenzelburger, 2015). Del mismo modo, aunque la situación legal en los países occidentales ha cambiado a favor de las personas homosexuales, la situación social parece que realmente no lo ha hecho, ya que no existe una total aceptación de la homosexualidad y esto hace que personas homosexuales aún no acepten su orientación sexual y vivan sus relaciones sexuales desde la ocultación.

Smith (1971) definió el término homofobia como una actitud de miedo y rechazo que las personas, especialmente heterosexuales, muestran hacia los homosexuales. A día de hoy, con independencia de que la legalidad vigente sea más o menos igualitaria, podemos considerar igual de válida esta definición que propuso este autor en los años setenta.

Su evolución ha seguido el mismo camino que los estudios sobre el racismo y el sexismo, englobando desde aquellas actitudes discriminatorias más hostiles y explícitas, tales como la agresión verbal, física o psicológica (homofobia manifiesta), hasta aquellas otras más latentes y sutiles, tales como los discursos culturales sobre el carácter patológico de la homosexualidad o la incapacitación de las parejas homosexuales para la adopción (homofobia sutil) (Quiles, Rodríguez y Torres, 2003). Debido a la mayor visibilidad del colectivo homosexual y a su larga trayectoria de lucha por la consecución de sus derechos, la homofobia ha evolucionado hacia expresiones más sutiles (Lameiras, Carrera y Rodríguez, 2013).

Además de estos dos tipos de homofobia que ejercen los demás hacia la persona homosexual, podemos hablar de aquella que la propia persona se ejerce a sí misma (homofobia internalizada). Este término engloba aquellas actitudes negativas que los individuos pueden tener hacia la propia homosexualidad (Mayfield, 2001). Por tanto, aunque parece que la sociedad ha avanzado respecto a la aceptación de la homosexualidad, siguen estando presentes ciertas actitudes homófobas, en los demás o en uno mismo, que parecen ejercer de mediadores en cuanto a la aceptación tanto de la propia orientación homosexual como de la realización de prácticas sexuales homosexuales.

Todas estas actitudes homófobas conllevan repercusiones tanto físicas como psicológicas sobre las personas que se definen como no heterosexuales. De hecho, diversos estudios demuestran que las personas lesbianas, bisexuales y gays presentan mayores niveles de depresión y ansiedad y bajos niveles de autoestima (Espada, Morales, Orgilés y Ballester, 2012), además de experimentar niveles significativamente más altos de ideación suicida que los heterosexuales (Baiocco, loverno, Lonigro, Baumgartner y Laghi, 2015).

Estos niveles elevados de depresión, ansiedad e ideación suicida entre el colectivo LGB se deben, en parte, al estigma social asociado a las actitudes de prejuicio y discriminación hacia estas orientaciones sexuales minoritarias que ejerce de mediador en su calidad de vida sexual (Baiocco y cols., 2015).

Atendiendo a la importancia que supone experimentar una vivencia favorable de la propia sexualidad para la calidad de vida de los individuos, el presente estudio se plantea como objetivo analizar el grado de aceptación en la definición de la propia orientación sexual y la 
flexibilidad en la realización de distintos comportamientos sexuales que se pueden alejar o no de lo que se establece como propio de esa orientación sexual definida, especialmente en una etapa de la vida tan complicada como es la adolescencia.

\section{Método}

\section{Participantes}

En esta investigación participaron un total de 400 alumnos/as (50,5\% chicas), con edades comprendidas entre 14 y 18 años ( $M=16,39$; DT $\pm 1,12$ ), que se encontraban escolarizados en diferentes centros de enseñanza de secundaria de las ciudades de Valencia y Castellón de la Plana (Comunidad Valenciana).

\section{Instrumentos}

Para llevar a cabo este estudio, los participantes cumplimentaron el cuestionario de intereses y prácticas sexuales (Unisexsida, 2014).

Este cuestionario consta de cinco ítems que evalúan la orientación sexual tanto de forma categorial como continua y permite obtener información acerca de deseos, fantasías y relaciones sexuales que presentan los adolescentes con personas de su mismo o diferente sexo, además de su disposición a realizar determinadas prácticas sexuales homosexuales.

En esta investigación se presentan únicamente los resultados de dos ítems: autodefinición de la orientación sexual, recogida de manera categórica (homosexual, bisexual, heterosexual), y disposición hacia la realización de distintas prácticas sexuales con personas del mismo sexo (besos en los labios, abrazos y caricias desnudos, masturbar a la otra persona, dejar que la otra persona te masturbe, sexo oral a la otra persona y dejar que la otra persona te haga sexo oral), recogida mediante un formato con tres opciones de respuesta (de ninguna manera, solo si la otra persona es atractiva o fácilmente).

\section{Procedimiento}

El procedimiento para poder llevar a cabo esta investigación constó de varios pasos. En primer lugar, se seleccionaron los centros de educación secundaria donde se pretendía obtener la muestra. En segundo lugar, se contactó con el director o la directora de cada centro y con la AMPA (Asociación de Madres y Padres de Alumnos), enfatizando la necesidad de su colaboración, con el fin de que autorizaran la administración del cuestionario ya que el estudio se iba a llevar a cabo con menores de edad. En tercer lugar, una vez obtenido el consentimiento informado por parte del director o la directora y de la AMPA, se pidió autorización al tutor/a de los grupos y se acordó con el profesorado un horario de administración. Por último, se acudió al centro en el horario acordado y se administró el cuestionario al alumnado, explicándoles en qué consistía nuestra investigación y enfatizando la participación voluntaria y anónima. 


\section{Análisis estadístico}

Para llevar a cabo los análisis estadísticos se utilizó el paquete SPSS para Windows (versión 22.0). En primer lugar, se realizaron los análisis descriptivos para explicar las características de la población objeto de estudio, obteniendo la media y desviación típica de la edad y el porcentaje de participantes de cada sexo. Al tratarse de variables categoriales, se utilizó la prueba Chi-cuadrado para observar las diferencias significativas en función del sexo, la orientación sexual y la disposición a realizar determinadas prácticas sexuales homosexuales.

\section{Resultados}

Los resultados muestran que un $89,2 \%$ de la población adolescente se define como heterosexual, un 6,2 \% como bisexual y un 4,5\% como homosexual. En cuanto a la definición de la propia orientación sexual (véase la tabla 1), no se encuentran diferencias significativas $\left(x^{2}=2,812 ; p=0,245\right)$. En el caso de los chicos, un $89,9 \%$ se identifican como heterosexuales, un $4,5 \%$ como bisexuales y un $5,6 \%$ como homosexuales. En el caso de las chicas, se observa cómo un $88,6 \%$ se identifican como heterosexuales, un 7,9\% como bisexuales y un $3,5 \%$ como homosexuales. Por tanto, el porcentaje de participantes que se define en alguna orientación distinta a la heterosexual asciende hasta un $10,7 \%$.

Tabla 1

Autodefinición de la orientación sexual

\begin{tabular}{llllr}
\hline & & \multicolumn{3}{c}{ Sexo } \\
& $\begin{array}{c}\text { Chicos } \\
\%(n)\end{array}$ & $\begin{array}{c}\text { Chicas } \\
\%(n)\end{array}$ & Total \\
\hline \multirow{3}{*}{ Orientación sexual } & Heterosexual & $89,9(178)$ & $88,6(179)$ & $89,2(357)$ \\
& Bisexual & $4,5(9)$ & $7,9(16)$ & $6,2(25)$ \\
& Homosexual & $5,6(11)$ & $3,5(7)$ & $4,5(18)$ \\
\hline
\end{tabular}

En cuanto a la disposición a realizar distintas prácticas sexuales con personas del mismo sexo (véase la tabla 2), se encuentran diferencias significativas entre chicos y chicas a la hora de dar besos en los labios $\left(x^{2}=64,632 ; p<0,001\right)$ y abrazos y caricias desnudos $\left(x^{2}=15,181\right.$; $p<0,001)$, siendo las chicas las más dispuestas.

Respecto a la primera conducta, un $81,8 \%$ de los chicos afirma que no lo haría nunca, un $3 \%$ solo si la otra persona les resultara atractiva y un $15,2 \%$ lo haría fácilmente. Así mismo, un $43,6 \%$ de las chicas no lo haría nunca, frente a un $17,8 \%$ que lo haría en caso de sentirse atraída por la otra persona y un 38,6\% que lo haría fácilmente.

Por otra parte, cuando se trata de dar abrazos y caricias desnudos a otra persona del mismo sexo, del total de chicos, un $87,4 \%$ no lo haría nunca, un 2,5\% lo haría únicamente si sintieran atracción por la otra persona y un $10,1 \%$ lo haría fácilmente. Por lo que respecta a las chicas, un $72,3 \%$ dice que no lo haría nunca, mientras que un $8,9 \%$ lo haría en el caso de que la otra persona les resultara atractiva y un $18,8 \%$ lo haría fácilmente. 
Tabla 2

Diferencias entre chicos y chicas en la disposición a realizar distintas prácticas sexuales con personas del mismo sexo

\begin{tabular}{|c|c|c|c|c|}
\hline & \multicolumn{2}{|c|}{ Sexo } & \multirow[b]{2}{*}{$\chi^{2}$} & \multirow[b]{2}{*}{$p$} \\
\hline & $\begin{array}{l}\text { Chicos } \\
\%(n)\end{array}$ & $\begin{array}{l}\text { Chicas } \\
\%(n)\end{array}$ & & \\
\hline \multicolumn{5}{|l|}{ Besos en los labios } \\
\hline De ninguna manera & $81,8(162)$ & $43,6(88)$ & & \\
\hline Solo si la otra persona es atractiva & $3(6)$ & $17,8(36)$ & 64,63 & $<0,001$ \\
\hline Fácilmente & $15,2(30)$ & $38,6(78)$ & & \\
\hline \multicolumn{5}{|l|}{ Abrazos y caricias desnudos } \\
\hline De ninguna manera & $87,4(173)$ & $72,3(146)$ & & \\
\hline Solo si la otra persona es atractiva & $2,5(5)$ & $8,9(18)$ & 15,18 & $<0,001$ \\
\hline Fácilmente & $10,1(20)$ & $18,8(38)$ & & \\
\hline \multicolumn{5}{|l|}{$\begin{array}{l}\text { Masturbar a otra persona del mismo } \\
\text { sexo }\end{array}$} \\
\hline De ninguna manera & $88,9(176)$ & $83,2(168)$ & & \\
\hline Solo si la otra persona es atractiva & $2,5(5)$ & $5,9(12)$ & 3,67 & $<0,160$ \\
\hline Fácilmente & $8,6(17)$ & $10,9(22)$ & & \\
\hline \multicolumn{5}{|l|}{$\begin{array}{l}\text { Dejarme masturbar por una persona } \\
\text { del mismo sexo }\end{array}$} \\
\hline De ninguna manera & $87,4(173)$ & $81,7(165)$ & & \\
\hline Solo si la otra persona es atractiva & $2,5(5)$ & $6,9(14)$ & 4,62 & $<0,099$ \\
\hline Fácilmente & $10,1(20)$ & $11,4(23)$ & & \\
\hline \multicolumn{5}{|l|}{$\begin{array}{l}\text { Practicar sexo oral a una persona del } \\
\text { mismo sexo }\end{array}$} \\
\hline De ninguna manera & $88,9(176)$ & $87,1(176)$ & & \\
\hline Solo si la otra persona es atractiva & $3,5(7)$ & $3(6)$ & 0,75 & $<0,687$ \\
\hline Fácilmente & $7,6(15)$ & $9,9(20)$ & & \\
\hline \multicolumn{5}{|l|}{$\begin{array}{l}\text { Recibir sexo oral de una persona del } \\
\text { mismo sexo }\end{array}$} \\
\hline De ninguna manera & $87,9(174)$ & $83,6(168)$ & & \\
\hline Solo si la otra persona es atractiva & $2,5(5)$ & $4(8)$ & 1,593 & $<0,451$ \\
\hline Fácilmente & $9,6(19)$ & $12,4(25)$ & & \\
\hline
\end{tabular}

En relación a la disposición a realizar distintas prácticas sexuales con personas del mismo sexo entre las distintas orientaciones sexuales (véase la tabla 3), se encuentran diferencias significativas en la disposición a realizar todas la prácticas sexuales descritas con otra persona del mismo sexo. Como parece lógico, los adolescentes que se definen como heterosexuales presentan menor disposición a realizar cualquiera de las prácticas sexuales homosexuales en comparación con los bisexuales u homosexuales. 
Tabla 3

Diferencias entre orientaciones sexuales en la disposición a realizar distintas prácticas sexuales con personas del mismo sexo

\begin{tabular}{|c|c|c|c|c|c|}
\hline & \multicolumn{3}{|c|}{ Orientación sexual } & \multirow[b]{2}{*}{$\chi^{2}$} & \multirow[b]{2}{*}{$p$} \\
\hline & $\begin{array}{l}\text { Heterosexual } \\
\text { \%(n) }\end{array}$ & $\begin{array}{l}\text { Bisexual } \\
\%(n)\end{array}$ & $\begin{array}{l}\text { Homo- } \\
\text { sexual }\end{array}$ & & \\
\hline \multicolumn{6}{|l|}{ Besos en los labios } \\
\hline De ninguna manera & $70(250)$ & $0(0)$ & $0(0)$ & & \\
\hline $\begin{array}{l}\text { Solo si la otra persona es } \\
\text { atractiva }\end{array}$ & $11,2(40)$ & $8(2)$ & $0(0)$ & 115,93 & $<0,001$ \\
\hline Fácilmente & $18,8(67)$ & $92(23)$ & $100(18)$ & & \\
\hline \multicolumn{6}{|l|}{ Abrazos y caricias desnudos } \\
\hline De ninguna manera & $89,4(319)$ & $0(0)$ & $0(0)$ & & \\
\hline $\begin{array}{l}\text { Solo si la otra persona es } \\
\text { atractiva }\end{array}$ & $5(18)$ & $12(3)$ & $11,1(2)$ & 222,66 & $<0,001$ \\
\hline Fácilmente & $5,6(20)$ & $88(22)$ & $88,9(16)$ & & \\
\hline \multicolumn{6}{|l|}{$\begin{array}{l}\text { Masturbar a otra persona del } \\
\text { mismo sexo }\end{array}$} \\
\hline De ninguna manera & $95,8(342)$ & $8(2)$ & $0(0)$ & & \\
\hline $\begin{array}{l}\text { Solo si la otra persona es } \\
\text { atractiva }\end{array}$ & $3,4(12)$ & $12(3)$ & $11,1(2)$ & 314,57 & $<0,001$ \\
\hline Fácilmente & $0,8(3)$ & $80(20)$ & $88,9(16)$ & & \\
\hline \multicolumn{6}{|l|}{$\begin{array}{l}\text { Dejarme masturbar por una } \\
\text { persona del mismo sexo }\end{array}$} \\
\hline De ninguna manera & $94,4(337)$ & $4(1)$ & $0(0)$ & & \\
\hline $\begin{array}{l}\text { Solo si la otra persona es } \\
\text { atractiva }\end{array}$ & $3,9(14)$ & $12(3)$ & $11,1(2)$ & 297,67 & $<0,001$ \\
\hline Fácilmente & $1,7(6)$ & $84(21)$ & $88,9(16)$ & & \\
\hline \multicolumn{6}{|l|}{$\begin{array}{l}\text { Practicar sexo oral a una } \\
\text { persona del mismo sexo }\end{array}$} \\
\hline De ninguna manera & $97,8(349)$ & $12(3)$ & $0(0)$ & & \\
\hline $\begin{array}{l}\text { Solo si la otra persona es } \\
\text { atractiva }\end{array}$ & $2(7)$ & $16(4)$ & $11,1(2)$ & 329,55 & $<0,001$ \\
\hline Fácilmente & $0,3(1)$ & $72(18)$ & $88,9(16)$ & & \\
\hline \multicolumn{6}{|l|}{$\begin{array}{l}\text { Recibir sexo oral de una } \\
\text { persona del mismo sexo }\end{array}$} \\
\hline De ninguna manera & $96,1(342)$ & $0(0)$ & $0(0)$ & & \\
\hline $\begin{array}{l}\text { Solo si la otra persona es } \\
\text { atractiva }\end{array}$ & $2,5(9)$ & $8(2)$ & $11,1(2)$ & 324,51 & $<0,001$ \\
\hline Fácilmente & $1,4(5)$ & $92(23)$ & $88,9(16)$ & & \\
\hline
\end{tabular}


Por último, respecto a la disposición a realizar distintas prácticas sexuales con personas del mismo sexo en el grupo de adolescentes que se consideran heterosexuales, se observa que entre un $4,5 \%$ y un $59,9 \%$ se encuentran dispuestos a realizar ciertas prácticas con otra persona de su mismo sexo. Así mismo, existen diferencias significativas entre chicos y chicas respecto a dar besos en los labios $\left(x^{2}=74,626, p<0,000\right)$, abrazos y caricias desnudos $\left(x^{2}=22,972, p<0,000\right)$ y dejarse masturbar por otra persona de su mismo sexo $\left(x^{2}=7,380\right.$, $p<0,05)$, siendo las chicas las que muestran una menor reticencia especialmente si la otra persona les resulta atractiva (véase la tabla 4).

Tabla 4

Diferencias entre chicos y chicas heterosexuales en la disposición a realizar distintas prácticas sexuales con personas del mismo sexo

\begin{tabular}{|c|c|c|c|c|}
\hline & \multicolumn{2}{|c|}{ Sexo } & \multirow[b]{2}{*}{$\chi^{2}$} & \multirow[b]{2}{*}{$\mathrm{p}$} \\
\hline & $\begin{array}{l}\text { Chicos } \\
\%(n)\end{array}$ & $\begin{array}{l}\text { Chicas } \\
\%(n)\end{array}$ & & \\
\hline \multicolumn{5}{|l|}{ Besos en los labios } \\
\hline De ninguna manera & $91(162)$ & $49,2(88)$ & & \\
\hline Solo si la otra persona es atractiva & $2,8(5)$ & $19,6(35)$ & 74,63 & $<0,001$ \\
\hline Fácilmente & $6,2(11)$ & $31,3(56)$ & & \\
\hline \multicolumn{5}{|l|}{ Abrazos y caricias desnudos } \\
\hline De ninguna manera & $97,2(173)$ & $81,6(146)$ & & \\
\hline Solo si la otra persona es atractiva & $1,1(2)$ & $8,9(16)$ & 22,97 & $<0,001$ \\
\hline Fácilmente & $1,7(3)$ & $9,5(17)$ & & \\
\hline \multicolumn{5}{|l|}{$\begin{array}{l}\text { Masturbar a otra persona del mismo } \\
\text { sexo }\end{array}$} \\
\hline De ninguna manera & $98,3(175)$ & $93,3(167)$ & & \\
\hline Solo si la otra persona es atractiva & $1,1(2)$ & $5,6(10)$ & 5,85 & $<0,054$ \\
\hline Fácilmente & $0,6(1)$ & $1,1(2)$ & & \\
\hline \multicolumn{5}{|l|}{$\begin{array}{l}\text { Dejarme masturbar por una persona } \\
\text { del mismo sexo }\end{array}$} \\
\hline De ninguna manera & $97,2(173)$ & $91,6(164)$ & & \\
\hline Solo si la otra persona es atractiva & $1,1(2)$ & $6,7(12)$ & 7,38 & $<0,05$ \\
\hline Fácilmente & $1,7(3)$ & $1,7(3)$ & & \\
\hline \multicolumn{5}{|l|}{$\begin{array}{l}\text { Practicar sexo oral a una persona del } \\
\text { mismo sexo }\end{array}$} \\
\hline De ninguna manera & $98,3(175)$ & $97,2(1764)$ & & \\
\hline Solo si la otra persona es atractiva & $1,7(3)$ & $2,2(4)$ & 1,14 & $<0,565$ \\
\hline Fácilmente & $0(0)$ & $0,6(1)$ & & \\
\hline
\end{tabular}




\begin{tabular}{lllll}
\hline & $\begin{array}{c}\text { Chicos } \\
\%(\mathrm{n})\end{array}$ & $\begin{array}{c}\text { Chicas } \\
\%(\mathrm{n})\end{array}$ & $\chi^{2}$ & $\mathrm{p}$ \\
\hline $\begin{array}{l}\text { Recibir sexo oral de una persona del } \\
\text { mismo sexo }\end{array}$ & & & & \\
$\quad$ De ninguna manera & $97,8(174)$ & $94,4(168)$ & & \\
$\quad$ Solo si la otra persona es atractiva & $1,1(2)$ & $3,9(7)$ & 3,08 & $<0,214$ \\
Fácilmente & $1,1(2)$ & $1,7(3)$ & & \\
\hline
\end{tabular}

\section{Discusión y conclusiones}

El objetivo de este estudio ha sido explorar la diversidad en la orientación sexual de los adolescentes españoles y su disposición a realizar distintas prácticas sexuales con personas de su mismo sexo.

Realizar una investigación de tal índole nos aporta obtener una información más amplia sobre la salud sexual de la población adolescente, que se considera de más difícil acceso porque requiere autorización por parte del centro docente y de los tutores legales, con el fin de detectar necesidades y desarrollar programas preventivos que reflejen la no consideración de la orientación sexual como una categoría única y permitan el desarrollo de actitudes más positivas hacia la diversidad afectivo-sexual.

De manera general, los resultados indican que un $89,2 \%$ de la población adolescente se define como heterosexual, un 6,2 \% como bisexual y un 4,5\% como homosexual. Por tanto, el porcentaje de adolescentes homosexuales y bisexuales representan un 10,7 \% de los participantes. Estos resultados van en la misma línea que los obtenidos en otros estudios realizados en población adolescente y adulta, en los que la prevalencia de orientaciones sexuales distintas a la heterosexualidad se sitúa en torno al 5-10 \% (Sell, Wells y Wypij, 1995). Debido a que en la adolescencia es cuando se va desarrollando una orientación sexual definida, cabía esperar que los porcentajes obtenidos fueran algo mayores a los obtenidos únicamente en población adulta, ya que en esta etapa evolutiva no se encuentra totalmente definida y se presentan sentimientos de inseguridad y ambivalencia (Monroy, 2002), aunque resultados contrarios también son explicables por la falta de autoconocimiento sexual que puede darse a estas edades y el temor a etiquetarse de un modo no aceptado socialmente.

Respecto a las diferencias entre sexos, los resultados indican que las chicas adolescentes presentan mayor disposición a realizar prácticas sexuales homosexuales que los chicos. Esto es especialmente evidente en cuanto a dar besos en los labios y abrazos y caricias desnudos. En este sentido, observamos diferencias con los estudios realizados en la década de los 90, que encontraron una mayor prevalencia de experiencias homosexuales en chicos que en chicas (Sell y cols., 1995). Estas diferencias pueden deberse a que, actualmente, vivimos en una sociedad donde aparentemente existe una mayor aceptación de la homosexualidad y, debido a ello, ha aumentado el porcentaje de adolescentes que admiten haber tenido alguna atracción homosexual o la disposición a realizar prácticas homosexuales, que es mayor en el caso de las chicas porque presentan mayor flexibilidad en su orientación sexual autodefinida (De la Rubia, 2011).

Los resultados también indican, como era de esperar, que los adolescentes que se identifican como heterosexuales presentan una disposición más baja a realizar prácticas sexuales homosexuales que los bisexuales u homosexuales. Sin embargo, resulta muy notable que en- 
tre un $4,5 \%$ y un $59,9 \%$ de estos adolescentes que se consideran heterosexuales se encuentran dispuestos a realizar ciertas prácticas sexuales con otra persona de su mismo sexo. Así mismo, si observamos las diferencias entre sexos, las chicas muestran una mayor disposición a dar besos en los labios, abrazos y caricias desnudas y dejarse masturbar por otra persona de su mismo sexo. Este hecho puede deberse a que los chicos heterosexuales presentan mayores actitudes homófobas y, por tanto, rechazan de manera más rotunda la realización de cualquier comportamiento homosexual (Carrera-Fernández, Lameiras-Fernández, RodríguezCastro y Vallejo-Medina, 2013).

Las principales limitaciones encontradas en este estudio han sido, por una parte, la falta de colaboración por parte de algunos centros educativos, que se encuentra justificada en muchos casos por el rechazo que manifiesta la sociedad en cuanto a la realización de estudios de tal índole con población de menores; por otra parte, la falta de participantes del ámbito rural, que ayuden a identificar las necesidades específicas de su entorno e implementar programas de prevención de la homofobia y de salud psicosexual adaptados a su realidad particular.

En vista a futuras líneas de investigación sería aconsejable ampliar el total de participantes tanto en tamaño como en rango de edades con el objetivo de obtener una información más amplia sobre el desarrollo de la orientación sexual a lo largo de distintas etapas del ciclo evolutivo y acerca de cómo influyen los agentes de socialización en el desarrollo de actitudes hacia la diversidad afectivo-sexual.

En conclusión, en nuestro estudio con población adolescente española encontramos que, cuando se pregunta por la orientación sexual de manera categórica (heterosexual, bisexual, homosexual), un porcentaje bajo de participantes se definen como bisexuales u homosexuales. Sin embargo, existen participantes que, aun reconociéndose como heterosexuales, podrían presentar algunos patrones de comportamiento sexual homosexuales. Por último, indistintamente de la orientación sexual definida, las chicas presentan mayor disposición a realizar prácticas sexuales homosexuales que los chicos.

\section{Referencias bibliográficas}

American Psychiatric Association (2013). Diagnosticand Statistical Manual of Mental Disorders (5. ${ }^{a}$ edición). Washington: APA.

Baiocco, R., loverno, S., Lonigro, A., Baumgartner, E. y Laghi, F. (2015). Suicidal Ideation among Italian and Spanish Young Adults: The Role of Sexual Orientation. Archives of suicide research, 19, 75-88.

Carrera-Fernández, M. V., Lameiras-Fernández, M., Rodríguez-Castro, Y. y Vallejo-Medina, P. (2013). Bullying among Spanish secondary education students: the role of gender traits, sexism, and homophobia. Journal of interpersonal violence, 28, 2915-2940.

De la Rubia, J. M. (2011). Orientación sexual en adolescentes y jóvenes mexicanos de 12 a 29 años de edad. Psicología desde el Caribe, 27, 112-135.

Espada, J. P., Morales, A., Orgilés, M. y Ballester, R. (2012). Autoconcepto, ansiedad social y sintomatología depresiva en adolescentes españoles según su orientación sexual. Ansiedad y Estrés, 18, 31-41.

Grant, J. M., Mottet, L., Tanis, J. E., Harrison, J., Herman, J. y Keisling, M. (2011). Injustice at every turn: A report of the National Transgender Discrimination Survey. National Center for Transgender Equality.

Jäckle, S. y Wenzelburger, G. (2015). Religion, religiosity, and the attitudes toward homosexuality -a multilevel analysis of 79 countries. Journal of homosexuality, 62, 207-241. 
Lameiras, M., Carrera, M. V. y Rodríguez, Y. (2013). Sexualidad y salud. El estudio de la sexualidad humana desde una perspectiva de género. Vigo: Servizo de Publicacións da Universidade de Vigo.

Mayfield W. (2001).The development of an internalized homonegativity inventory for gay men. Journal of Homosexuality, 41, 53-76.

Monroy, A. (2002). Salud y sexualidad en la adolescencia y juventud. Editorial Pax México.

Quiles, M. N., Rodríguez, V. B. y Torres, R. R. (2003). La medida de la homofobia manifiesta y sutil. Psicothema, 15, 197-204.

Sell, R. L., Wells, J. A. y Wypij, D. (1995). The Prevalence of Homosexual Behavior and Attraction in the United States, the United Kingdom and France: Results of National PopulationBased Samples. Archives of Sexual Behavior, 24, 234-248. 